\title{
IncRNA-NRF is a Potential Biomarker of Heart Failure After Acute Myocardial Infarction
}

\author{
Li Yan ${ }^{1,2} \cdot$ Yu Zhang ${ }^{3} \cdot$ Wei Zhang ${ }^{2} \cdot$ Sheng-Qiong Deng ${ }^{4} \cdot$ Zhi-Ru Ge $^{2}$ \\ Received: 5 November 2019 / Accepted: 12 May 2020 / Published online: 21 May 2020 \\ (C) The Author(s) 2020
}

\begin{abstract}
Long non-coding RNAs (lncRNAs) are a new focus in cardiovascular diseases. The necrosis-related factor (NRF) is a newly discovered lncRNA, which is increased in myocardial injury. We investigated the role of lncRNA-NRF in heart failure (HF) after acute myocardial infarction (AMI) to find a biomarker for early HF detection. This was a cross-sectional study of 76 AMI patients with HF and 58 AMI patients without HF. IncRNA-NRF was shown to be increased in AMI patients with HF compared with AMI patients without $\mathrm{HF}$ and had predictive value for diagnosis of HF. It had a high diagnostic value for HF (AUC, 0.975), while the AUC for N-terminal pro-brain natriuretic peptide was 0.720 . Our findings suggest that lncRNA-NRF may represent a marker of risk for development of HF post-AMI.

Keywords Heart failure · Acute myocardial infarction . lncRNA-NRF · Diagnosis

$\begin{array}{ll}\text { eGFR } & \text { Estimated glomerular filtration rate } \\ \text { NT-proBNP } & \text { N-terminal pro-brain natriuretic peptide } \\ \text { LVEF } & \text { Left ventricular ejection fraction } \\ \text { LVFS } & \text { Left ventricular fractional shortening } \\ \text { LVEDD } & \text { Left ventricular end-diastolic dimension } \\ \text { TnI } & \text { Troponin I } \\ \text { TIMI } & \text { Thrombolysis in myocardial infarction }\end{array}$
\end{abstract}

$\begin{array}{ll}\text { Abbreviations } \\ \text { HF } & \text { Heart failure } \\ \text { AMI } & \text { Myocardial infarction } \\ \text { BMI } & \text { Body mass index } \\ \text { TG } & \text { Triglycerides } \\ \text { TC } & \text { Total cholesterol } \\ \text { LDL-C } & \text { Low-density lipoprotein cholesterol } \\ \text { HDL-C } & \text { High-density lipoprotein cholesterol } \\ \text { SCr } & \text { Serum creatinine }\end{array}$

Associate Editor Ana Barac oversaw the review of this article

Electronic supplementary material The online version of this article (https://doi.org/10.1007/s12265-020-10029-0) contains supplementary material, which is available to authorized users.

Zhi-Ru Ge

995097927@qq.com

1 Ningxia Medical University, Yinchuan 750000, China

2 Department of Cardiology, Shanghai Gongli Hospital, The Second Military Medical University, Shanghai 200135, China

3 Department of Emergency Medicine, Shanghai Gongli Hospital, The Second Military Medical University, Shanghai 200135, China

4 Department of Clinical Laboratory, Shanghai Gongli Hospital, The Second Military Medical University, Shanghai 200135, China

\section{Introduction}

Acute myocardial infarction (AMI), one of the most serious coronary artery diseases [1], and the heart failure (HF) that often follows are among the leading causes of death and disability worldwide [2-4]. One contributing factor is the existence of "myocardial reperfusion injuries" [4], whereby myocardial injury and cardiomyocyte death occur on the reperfusion of acutely ischemic myocardium because of a number of factors [5-7], including mitochondrial calcium overload, mitochondrial oxidative stress, ATP depletion $[5,7,8]$, and the opening of the mitochondrial permeability transition pore [6]. Medical therapy is the most effective treatment, but a lack of symptoms in the early stages leads to poor prognosis. Therefore, exploring effective biomarkers is of great importance for early detection and improving prognosis in $\mathrm{HF}$ after AMI.

Long non-coding RNAs (lncRNAs) are located in the cell nucleus or cytoplasm and have a length of more than 200 nucleotides $[9,10]$. Recently, studies have demonstrated that lncRNAs are involved in many complex cell processes in 
cardiovascular disease [11-13]. For example, the lncRNA necrosis-related factor (NRF) was confirmed to be increased in myocardial injury, and silencing of NRF increased miR873 expression and decreased levels of the miR-873 targets, receptor-interacting serine/threonine-protein kinase 1 (RIPK1) and RIPK3, which led to a sharp reduction in myocardial necrosis [14]. However, the role of lncRNA-NRF in the diagnosis of HF after AMI remains unclear. Here, we assessed the expression of IncRNA-NRF in patients with AMI and identified the diagnostic value of lncRNA-NRF for HF in AMI patients.

\section{Materials and methods}

\section{Participants}

The study was designed as a cross-sectional study and was conducted between June 2018 and February 2020 in the Department of Cardiology of Shanghai Gong Li Hospital. Patients with acute HF caused by serious arrhythmias, acute valve dysfunction, or other reasons, and those with chronic HF, pregnancy, cancer, acute infectious diseases, hepatic dysfunction, or abnormal renal function were excluded. A final population of 248 participants was enrolled in the study, and all patients who were diagnosed with AMI underwent percutaneous coronary intervention (PCI) as soon as possible. Based on diagnostic standards, the participants with HF post-PCI were divided into two groups of HF after AMI ( $n$ $=76)$ and non-HF after AMI $(n=58)$, and 30 basic clinical data-matched controls, 39 nonischemic HF patients, and 45 stable CAD patients. The AMI diagnosis was based on the 2017 ESC Guidelines for the management of AMI in patients presenting with ST-segment elevation [15]. At least one of the following was present in AMI patients: symptoms (i.e., persistent chest pain $>30 \mathrm{~min}$ ) and signs (i.e., 12-lead electrocardiogram, ST-segment elevation $>0.2 \mathrm{~mm}$ ) consistent with myocardial ischemia, at least a twofold increase in troponin I (TnI) [15]. The HF diagnosis was based on the 2016 ESC Guidelines for the diagnosis and treatment of acute and chronic HF [16]. At least one of the following was present in such patients: typical symptoms (dyspnea, fatigue), specific signs (elevated jugular venous pressure, hepatojugular reflux, pulmonary crackles, peripheral edema), age-specific levels of $\mathrm{N}$ terminal pro-brain natriuretic peptide (NT-proBNP) (ng/L) (age $<50$ years: $>450 \mathrm{ng} / \mathrm{L}$, age $50-75$ years: $>900 \mathrm{ng} / \mathrm{L}$, age $>75$ years: $>1800 \mathrm{ng} / \mathrm{L}$ ), and left ventricular ejection fraction $(\mathrm{LVEF})<50 \%$ [16]. Finally, 76 patients with $\mathrm{HF}$ after AMI were included in the study. All participants provided written informed consent before enrollment. The study was conducted in accordance with the principles contained within the Declaration of Helsinki.

\section{Anthropometric measurements}

Anthropometric determinations and blood extractions were performed on a single day. Height and weight were measured using calibrated portable electronic weighing scales and portable inflexible height measuring bars with barefoot participants wearing light indoor clothing. BMI was calculated using the standard BMI formula: body mass (in kilograms) divided by square of height (in meters).

\section{Blood sample preparation}

All blood samples were collected after participants had fasted overnight. Clinical variables included TnI, myoglobin, creatine kinase$\mathrm{MB}$, total cholesterol (TC), triglyceride (TG), low-density lipoprotein (LDL), high-density lipoprotein (HDL), serum creatinine (SCr), cystatin, urea nitrogen, and estimated glomerular filtration rate (eGFR). Serum TnI, CK-Mb, TG, TC, LDL-C, and HDL-C levels and other routine serum biochemical parameters were measured using a biochemical analyzer (Ortho-3160; Pencoed, Bridgend, UK). Levels of NT-proBNP and IncRNA-NRF were measured at immediate diagnosis (before $\mathrm{PCI}$ ), intraprocedural, and postoperatively at $4 \mathrm{~h}, 12 \mathrm{~h}, 24 \mathrm{~h}$, and $72 \mathrm{~h}$. Serum NTproBNP was measured using a biochemical analyzer (NanoChecker710; Nano-Ditech, Grandbury, American). All measurements were obtained using blinded quality control specimens at the Department of the Biochemical Laboratory at Shanghai Gong Li Hospital. Venous blood was obtained from 30 basic clinical data-matched controls, $39 \mathrm{HF}$ patients, and $45 \mathrm{CAD}$ patients. All blood samples for the lncRNA-NRF analysis were immediately placed into blood collection tubes containing EDTA, and were stored at $-80{ }^{\circ} \mathrm{C}$ before RNA extraction. The expression of lncRNA-NRF was detected through the RT-PCR analysis.

RNA extraction and RT-PCR analysis

Total RNA was isolated from venous blood samples using Trizol reagent (Invitrogen, Carlsbad, USA), and RNA quantification was performed using a Nanodrop (Thermo Scientific, Rockford, IL, USA). cDNA was synthesized using an Improm II reverse transcription kit (Promega, Madison, WI, USA) following the manufacturer's instructions. RT-PCR was performed using GAPDH as an internal control and SYBR Green to detect lncRNA-NRF levels, which were quantified through the $2^{-\Delta \Delta \mathrm{Ct}}$ method. The primers for the target genes (Table 1) were obtained from GenePharma (Shanghai, China).

\section{Statistical analysis}

Continuous variables are expressed as mean \pm standard deviations or median (interquartile range) and categorical variables as numeral (percentage). Independent Student's $t$ tests or one-way ANOVA for normal distribution and Wilcoxon rank-sum tests for asymmetric distribution were used to analyze the differences 
Table 1 Anthropometric and biochemical characteristics of the subjects

\begin{tabular}{|c|c|c|c|c|c|c|}
\hline & Control & HF post-AMI & Non-HF post-AMI & $\mathrm{HF}$ & CAD & $P$ value \\
\hline Male $(n, \%)$ & $13(43)$ & $42(55)$ & $33(57)$ & $19(49)$ & $22(49)$ & 0.712 \\
\hline Age (years) & 59.63. \pm 10.39 & $63.36 \pm 7.29$ & $62.84 \pm 11.12$ & $61.69 \pm 7.18$ & $64.36 \pm 7.68$ & 0.189 \\
\hline BMI (kg/m2) & $24.49 \pm 3.16$ & $23.91 \pm 2.76$ & $23.77 \pm 4.08$ & $24.05 \pm 4.04$ & $26.06 \pm 4.72$ & 0.019 \\
\hline Smoking history $(n, \%)$ & $10(33)$ & $43(57)$ & $22(38)$ & $10(26)$ & $13(29)$ & 0.060 \\
\hline Diabetic $(n, \%)$ & $9(30)$ & $30(39)$ & $17(28)$ & $11(28)$ & $14(31)$ & 0.663 \\
\hline Hypertensive $(n, \%)$ & $22(73)$ & $54(71)$ & $41(71)$ & $28(72)$ & $35(78)$ & 0.935 \\
\hline $\mathrm{TG}(\mathrm{mmol} / \mathrm{L})$ & $1.43 \pm 0.71$ & $1.36 \pm 1.10$ & $1.57 \pm 0.75$ & $1.07 \pm 0.54$ & $1.68 \pm 0.92$ & 0.016 \\
\hline $\mathrm{TC}(\mathrm{mmol} / \mathrm{L})$ & $4.41 \pm 1.16$ & $4.27 \pm 1.04$ & $4.50 \pm 1.07$ & $4.01 \pm 1.10$ & $4.42 \pm 1.50$ & 0.315 \\
\hline LDL-C (mmol/L) & $2.70 \pm 0.95$ & $2.78 \pm 0.93$ & $2.97 \pm 0.88$ & $2.41 \pm 1.00$ & $2.68 \pm 1.37$ & 0.127 \\
\hline $\mathrm{HDL}-\mathrm{C}(\mathrm{mmol} / \mathrm{L})$ & $1.08 \pm 0.23$ & $1.01 \pm 0.27$ & $1.00 \pm 0.45$ & $1.09 \pm 0.39$ & $1.04 \pm 0.34$ & 0.642 \\
\hline $\mathrm{SCr}(\mathrm{umol} / \mathrm{L})$ & $68(52,86)$ & $73(60,91)$ & $66(50,80)$ & $94(79,119)$ & $68(59,79)$ & 0.313 \\
\hline UN (umol/L) & $0.90(0.74,1.12)$ & $5.3(3.98,6.89)$ & $4.66(3.86,5.99)$ & $7.83(4.57,10.34)$ & $5.15(4.22,6.22)$ & 0.806 \\
\hline CysC (mg/L) & $5.22(4.16,7.44)$ & $1.11(0.93,1.37)$ & $0.81(0.74,1.04)$ & $1.17(0.96,1.50)$ & $0.93(0.81,1.14)$ & $<0.01^{* *}$ \\
\hline eGFR $\left(\mathrm{mL} / \mathrm{min} / 1.73 \mathrm{~m}^{2}\right)$ & $90(67,96)$ & $89(67,98)$ & $94(84.5,102)$ & $75(48,88)$ & $89(82,98)$ & 0.112 \\
\hline $\operatorname{LVEF}(\%)$ & $60.83 \pm 6.86$ & $55.59 \pm 8.71$ & $61.46 \pm 5.27$ & $54.61 \pm 8.53$ & $64.01 \pm 6.25$ & $<0.001 * * *$ \\
\hline LVFS $(\%)$ & $32.63 \pm 4.76$ & $29.31 \pm 5.55$ & $33.03 \pm 3.75$ & $29.01 \pm 5.89$ & $35.13 \pm 4.75$ & $<0.001 * * *$ \\
\hline LVEDD (mm) & $47.47 \pm 5.15$ & $47.89 \pm 5.74$ & $47.45 \pm 4.66$ & $52.03 \pm 8.98$ & $47.44 \pm 4.90$ & $<0.01 * *$ \\
\hline Postoperative NT-proBNP (ng/L) & $41(20,100)$ & $2136(1487,3461)$ & $249(151.5,442)$ & $2645(1903,3678)$ & $120(20,378)$ & $<0.001 * * *$ \\
\hline Intraprocedural NT-proBNP (ng/L) & 1 & $478(134,950)$ & $204(20.1,434.5)$ & 1 & 1 & $<0.001 * * *$ \\
\hline TnI & $0.02 \pm 0.03$ & $18.75 \pm 27.71$ & $11.28 \pm 21.94$ & $0.03 \pm 0.02$ & $0.26 \pm 1.30$ & $<0.001 * * *$ \\
\hline TIMI & I & $2.66 \pm 0.88$ & $2.64 \pm 0.77$ & / & I & 0.822 \\
\hline Number of branch lesions & I & $1.01 \pm 1.37$ & $1.17 \pm 1.40$ & / & I & 0.511 \\
\hline Immediate diagnosis of lncRNA-NRF & $0.95(0.87,1.02)$ & $1.71(1.21,2.02)$ & $0.56(0.48,0.61)$ & $1.07(0.93,1.29)$ & $0.67(0.59,0.86)$ & $<0.001 * * *$ \\
\hline Intraprocedural of lncRNA-NRF & 1 & $1.77(1.32,3.50)$ & $1.01(0.83,1.14)$ & / & / & $<0.001 * * *$ \\
\hline Postoperative $4 \mathrm{~h}$ of lncRNA-NRF & l & $1.18(0.87,1.63)$ & $0.66(0.56,0.74)$ & l & / & $<0.001 * * *$ \\
\hline Postoperative $12 \mathrm{~h}$ of $\operatorname{lncRNA-NRF}$ & / & $1.34(0.93,1.52)$ & $0.83(0.65,1.00)$ & l & / & $<0.001 * * *$ \\
\hline Postoperative $24 \mathrm{~h}$ of lncRNA-NRF & l & $1.07(0.98,1.47)$ & $0.98(0.86,1.06)$ & l & / & $<0.001 * * *$ \\
\hline Postoperative $72 \mathrm{~h}$ of $\operatorname{lncRNA-NRF}$ & / & $1.01(0.89,1.29)$ & $0.95(0.80,1.04)$ & l & / & $<0.01 * *$ \\
\hline
\end{tabular}

Results are expressed as mean \pm standard deviation, median (interquartile range) or $n(\%) . B M I$, body mass index; $T G$, triglycerides; $T C$, total cholesterol; $L D L-C$, low-density lipoprotein cholesterol; $H D L-C$, high-density lipoprotein cholesterol; $S C r$, serum creatinine; $C y s C$, cystatin; $U N$, urea nitrogen; $e G F R$, estimated glomerular filtration rate; $N T$-proBNP, N-terminal pro-brain natriuretic peptide; $L V E F$, left ventricular ejection fraction; $L V F S$, left ventricular fractional shortening; $L V E D D$, left ventricular end-diastolic dimension' TnI, troponin I; TIMI, thrombolysis in myocardial infarction

$* P<0.05, * * P<0.01, * * * P<0.001$

in continuous variables. Chi-squared tests and Fisher's exact tests were used to analyze categorical variables. The association between IncRNA-NRF and serum NT-proBNP was determined using Pearson correlation coefficient. The diagnostic value of lncRNA-NRF was estimated through building receiver operating characteristic (ROC) curves. A $P$ value $<0.05$ was considered statistically significant.

\section{Results}

\section{Baseline clinical characteristics of the study population}

This study included 76 AMI patients with HF post-PCI and 58 AMI patients without HF. The clinical characteristics of the study population are shown in Table 1 . There were no differences in age $(P=0.189)$, sex $(P=0.712)$, BMI $(P=0.019)$, medical history, presence of diabetes $(P=0.663)$, hypertension $(P=0.935)$, or smoking history $(P=0.060)$ between the five groups. There were significantly higher levels of CysC $(P$
$=0.001)$, but no significant differences in $\operatorname{SCr}(P=0.313)$, eGFR $(P=0.112)$, and urea nitrogen $(P=0.806)$ between the five groups. Remarkably, circulating lncRNA-NRF levels were significantly elevated in the HF group compared with the non-HF group after AMI $(P<0.001)$ (Table 1$)$.

\section{RT-PCR analysis of the expression of IncRNA-NRF}

RT-PCR analysis was used to measure the levels of circulating lncRNA-NRF in each group. The results demonstrated that the levels of circulating lncRNA-NRF were increased in patients with HF post-PCI compared with non-HF patients, reached a peak in intraprocedural and gradually returned to base line levels at 3 days (Fig. 1a, $P<0.001$ ). However, the levels of venous NT-proBNP were increased at 3 days after PCI in patients with HF post-PCI compared with controls (Fig. 1b, $P<0.001$ ). Circulating lncRNA-NRF levels expression showed higher in heart failure patients (Fig. 1c, $P=$ 0.128 ), and decreased in patients with stable CAD. (Fig. 1c, $P<0.05)$. 


\section{Pearson correlation coefficient of correlation between IncRNA-NRF and clinicopathological characteristics}

Associations between circulating lncRNA-NRF and serum NT-proBNP were examined using Pearson correlation coefficient. As shown in Table 2, circulating lncRNA-NRF levels were positively associated with serum NT-proBNP $\left(r^{2}=\right.$ $0.775, P<0.001)$ and TnI $\left(r^{2}=0.062, P<0.01\right)$ levels but negatively associated with $\operatorname{LVEF}\left(r^{2}=0.225, P<0.001\right)$ at the time of diagnosis. No associations with CK-Mb $\left(r^{2}=0.026, P\right.$ $=0.074)$ levels were observed. Moreover, subgroup analysis found that circulating lncRNA-NRF at the time of intraprocedural was not associated with coronary disease burden or TIMI flow (Table 1; Fig. 2; $P>0.05$ ) (Fig. 3).

\section{ROC curve analysis of the diagnostic value of IncRNA- NRF in HF after AMI}

Having established that lncRNA-NRF is present in the peripheral circulation and its levels are abnormally altered in HF patients after AMI, we sought to determine the potential utility
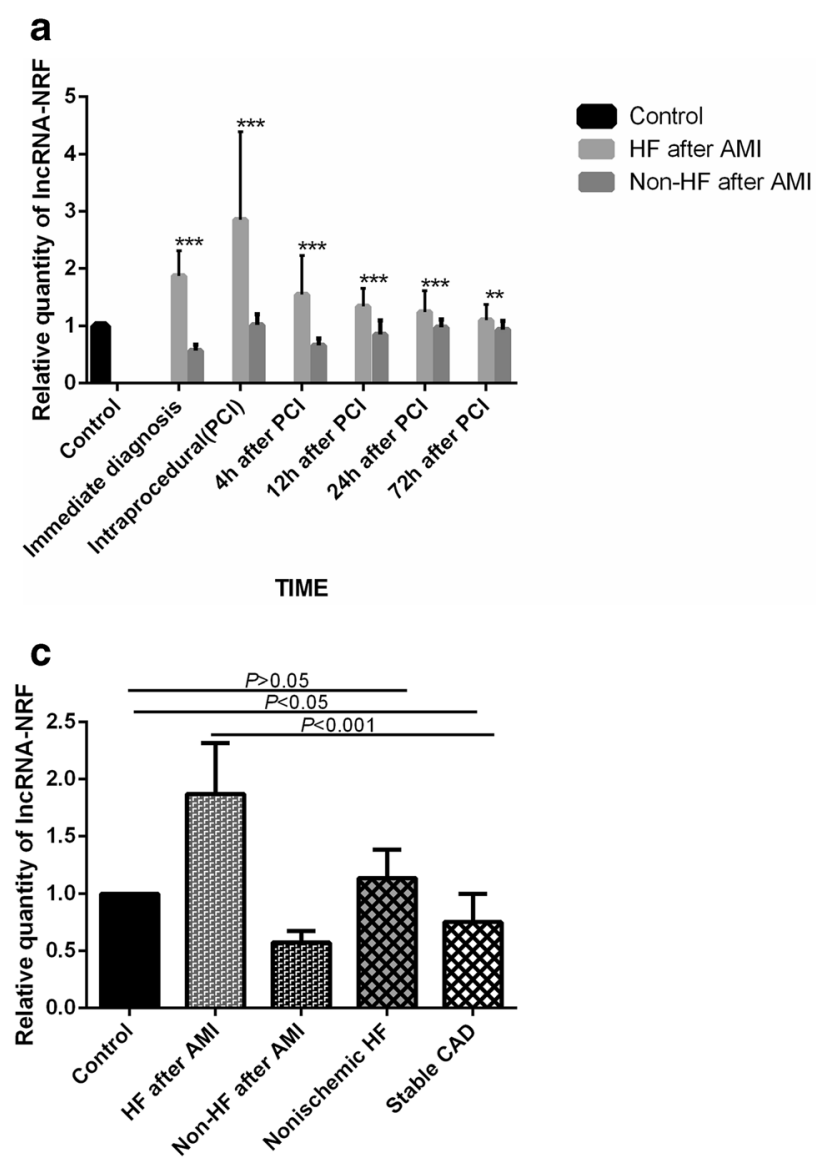

Fig. 1 Relative average lncRNA-NRF expression in all the patients. a Circulating lncRNA-NRF levels in AMI patients with HF and non-HF. b Venous blood NT-proBNP level in AMI patients with HF and non-HF. c of circulating lncRNA-NRF as a diagnostic biomarker for HF after AMI. To this end, ROC analysis was performed to evaluate the predictive power of circulating lncRNA-NRF at the time of diagnosis for HF after AMI. Our results showed that the area under the ROC curve was $0.975(95 \% \mathrm{CI}=0.952$ $0.998)$ for lncRNA-NRF, and $0.720(95 \% \mathrm{CI}=0.636-0.805)$ for NT-proBNP alone (Fig. 3).

\section{Discussion}

The development of heart failure is common during hospitalization in a setting of acute myocardial infarction, and this risk of death is greater than that of AMI alone [17, 18]. In contrast, the incidence characteristics of HF occurring after AMI is not known. Although advances in the management of AMI, including primary PCI strategies and sustained use of evidencebased treatments, are likely to have a beneficial impact on the apparent decline in mortality, post-AMI patients that survive AMI often develop ischemic HF [19]. Therefore, identifying biomarkers that predict the high risk of HF development in

\section{b}

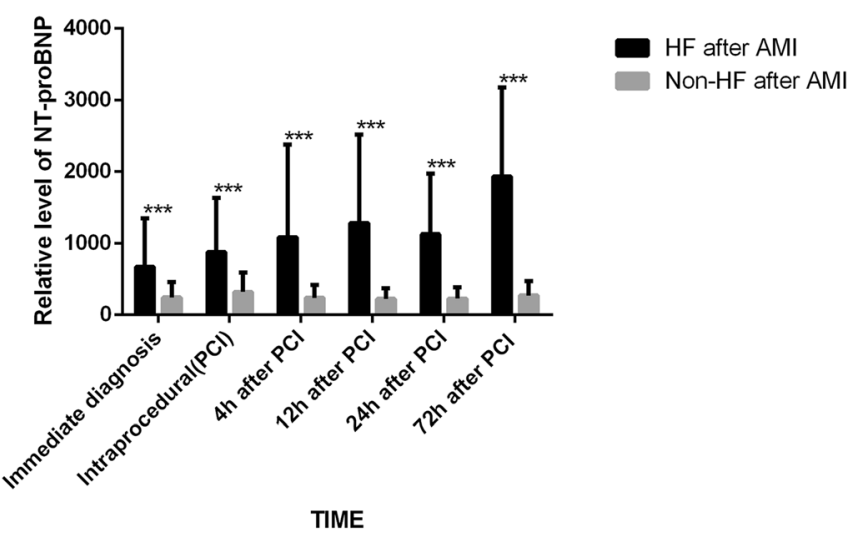

Circulating lncRNA-NRF levels in heart failure and stable CAD patients and at the time of diagnosis in patients with $\mathrm{HF}$ after AMI and non-HF after AMI. $* P<0.05, * * P<0.01, * * * P<0.001$ 
Table 2 Pearson correlation coefficient of correlations between lncRNA-NRF and clinicopathological characteristics

\begin{tabular}{|c|c|c|c|c|c|c|c|c|}
\hline & \multicolumn{2}{|l|}{ NT-proBNP } & \multicolumn{2}{|l|}{ LVEF } & \multicolumn{2}{|l|}{ TnI } & \multicolumn{2}{|l|}{$\mathrm{CK}-\mathrm{Mb}$} \\
\hline & OR (95\%CI) & $P$ value & OR $(95 \% \mathrm{CI})$ & $P$ value & OR $(95 \% \mathrm{CI})$ & $P$ value & OR $(95 \% \mathrm{CI})$ & $P$ value \\
\hline lncRNA-NRF & $r^{2}=0.775$ & $<0.001 * * *$ & $r^{2}=0.225$ & $<0.001 * * *$ & $r^{2}=0.062$ & $<0.01 * *$ & $r^{2}=0.026$ & 0.074 \\
\hline
\end{tabular}

Relationship between lncRNA-NRF levels and clinically relevant factors at the time of diagnosis. NT-proBNP, N-terminal pro-brain natriuretic peptide; $L V E F$, left ventricular ejection fraction; $T n I$, troponin I; $C K-M b$, creatine kinase-Mb

$* P<0.05, * * P<0.01, * * * P<0.001$

these patients post-AMI is needed to optimize treatment strategies to improve their long-term prognosis. To date, several biomarkers including NT-proBNP and troponin were reported to predict cardiovascular complications after AMI; nevertheless, whether these biomarkers predict future HF in patients after AMI is unclear, and their use is limited by the heterogeneity of genetic backgrounds, environment, biological behavior, and other diseases [20]. Accumulating evidence has shown that aberrantly regulated lncRNA is correlated with the progression of various diseases, like cardiovascular disease [21-23]. Although little is known about the origin and function of lncRNAs in circulation, their sensitive and stable differential expression in the blood of patients with cardiovascular diseases and healthy people makes them a potential biomarker [24]. The mechanism of action is likely that cardiac tissue damage leads to an additional release of IncRNAs, similar to the release of proteins. The progression to HF after AMI is primarily related to left ventricular remodeling, which is a heterogeneous process influenced by multiple factors, including microvascular dysfunction, infarct size, anterior infarct location, transmural extent of necrosis, and the perfusional status of the infarct-related artery [25].

Previous studies reported many virtual lncRNAs were associated with the development of HF after AMI. For instance, NRF was closely related to the necrotic death of cardiomyocytes by acting as an endogenous RNA sponge that
Fig. 2 Relationship between IncRNA-NRF levels and coronary disease burden at intraprocedural timepoint. a LncRNA-NRF expression levels in AMI patients with different coronary lesions. b LncRNANRF expression levels in AMI patients with different TIMI flow

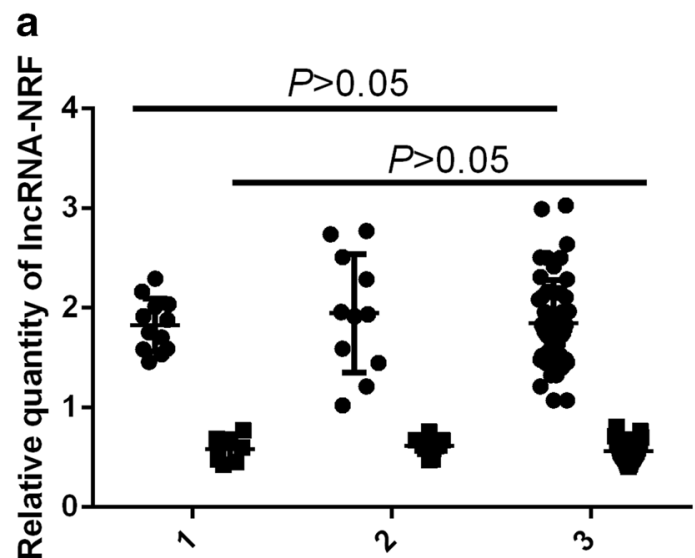

- HF after AMI

- Non-HF after AMI

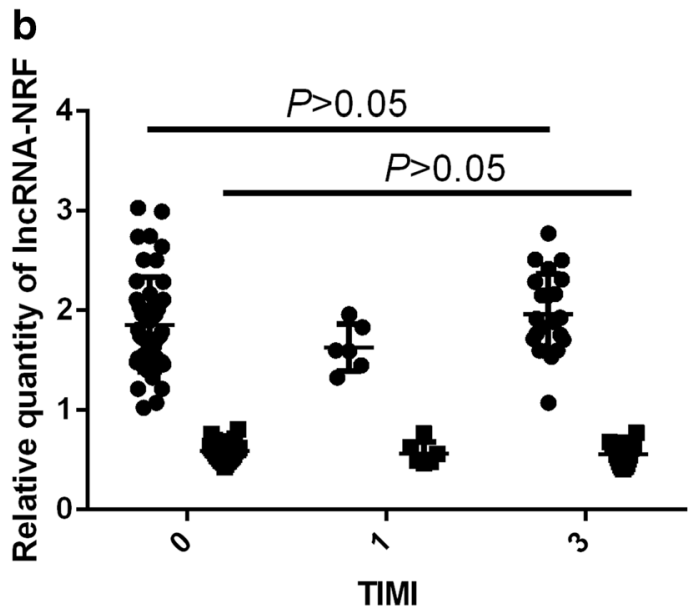

- HF after AMI

- Non-HF after AMI 
Fig. 3 ROC curve for patients with $\mathrm{HF}$ after AMI based on the expression of $\operatorname{lncRNA-NRF}$ and NT-proBNP at the time of diagnosis

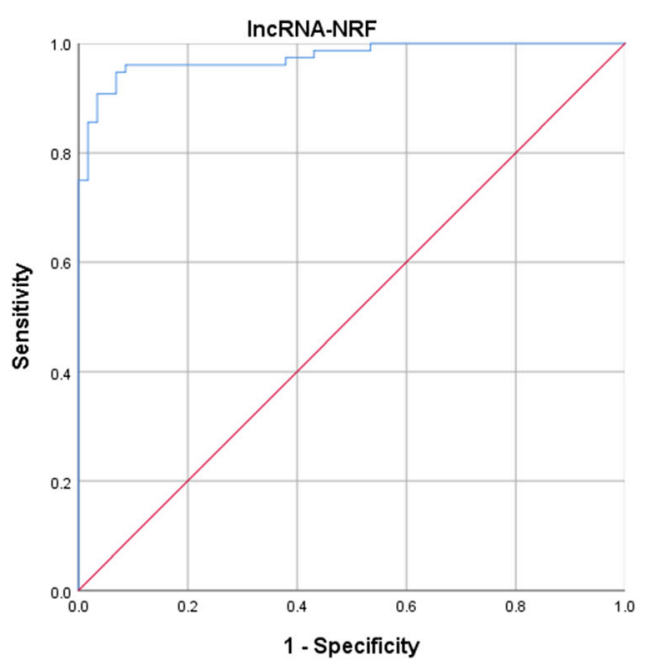

interacts with miR-873 in the cytoplasm [14]. Silencing of NRF increased miR-873 expression and decreased the levels of the miR-873 targets, RIPK1 and RIPK3, which led to a sharp reduction in myocardial necrosis [14]. A functional assay showed that silencing p53 reduced necrotic cell death and inhibited NRF promoter activity upon $\mathrm{H}_{2} \mathrm{O}_{2}$ treatment, and increased expression of miR-873 and decreased levels of RIPK1/RIPK3 were also induced by downregulated P53 levels [14]. Taken together, elucidating the regulatory relationships in the IncRNA-miRNA-mRNA axis during cardiomyocyte necrosis could shed light on potential therapeutic targets in cardiac dysfunction [12]. These results show that IncRNAs play important roles in the development and progression of HF. Substantial evidence from animal studies also suggests that NRF plays a significant role in HF [14]. However, because of a lack of clinical studies, little is known about whether the dynamic regulation of IncRNA-NRF can be viewed as a marker of disease development; however, the journey to fully explore the function of NRF in the regulation of cardiovascular physiology and pathology remains incomplete. Therefore, we concentrated on the biological behavior of LncRNA-NRF. In the current study, we first confirmed that AMI patients with $\mathrm{HF}$ and non-HF postPCI had different patterns of lncRNA-NRF expression in the peripheral blood: circulating lncRNA-NRF levels in AMI patients with HF post-PCI were significantly elevated compared with non-HF after AMI controls, reached a peak in intraprocedural and gradually returned to base line levels at 3 days. (Fig. $1 \mathrm{a}, P<0.01$ ). However, the levels of venous NT-proBNP were increased at 3 days after PCI in patients with HF postPCI compared with controls (Fig. 1b, $P<0.001$ ), far behind lncRNA-NRF. Several studies have recognized a consistent association between elevated troponin levels (using regular or high-sensitivity assays) and the risk of adverse clinical outcomes among patients with heart failure and reduced EF, even in the absence of intercurrent acute coronary events $[26,27]$. The present study also confirmed that circulating NRF levels are positively correlated with TnI levels and serum NT-proBNP but negatively associated with LVEF, no associations with $\mathrm{CK}-\mathrm{Mb}$ levels were observed, which is consistent with the conclusions reached using the Pearson correlation coefficient. Circulating NRF levels were positively associated with the severity of HF. We also investigated the clinical significance of IncRNA-NRF. Our results suggest that abnormal lncRNA-NRF expression is related to HF diagnosis. The high diagnostic value of lncRNANRF was demonstrated by a ROC curve with an AUC of 0.975 , and the ideal cutoff value was 0.952 (Fig. 2).

Care was taken to avoid bias in the current study. RT-PCR was performed in accordance with the manufacturer's instructions by a trained experimenter who was unaware of the identity of the experimental groups. Moreover, in the statistical analysis, adjustments were made for the confounding effects of risk factors for HF and circulating lncRNA-NRF levels. Finally, propensity score matching was used to reduce the effects of outcome-selection bias.

The current study has some limitations. First, it is a casecontrol study, which means that it can only show associations, not causality. Second, all of the patients with HF and some controls were administered drugs postoperatively. The effects of medication on IncRNA-NRF levels were not investigated in the current study. Third, the present study confirmed that circulating NRF levels were positively correlated with $\mathrm{TnI}$ levels. However, a study limitation was that samples of troponin were only collected at the time of diagnosis and $12 \mathrm{~h}$ after PCI; therefore, we could not determine a correlation between serial changes in troponin and serial changes in IncRNA-NRF. Fourth, contrary to expectations, HF occurred in the majority 
of patients with AMI. Therefore, we should expand the sample size in the future to further study the mechanism of HF and prognosis in such patients. Fifth, because all of the study participants were Chinese, the findings may not be generalizable to other ethnicities. Our findings should be confirmed in other populations.

\section{Conclusions}

lncRNA-NRF levels were significantly higher in Chinese post-AMI patients with HF compared with post-AMI controls. Furthermore, we demonstrated that lncRNA-NRF levels were positively associated with the severity of HF after AMI. However, the clinical application value and expression mechanism of NRF still need further study.

Acknowledgments We thank Conn Hastings, $\mathrm{PhD}$, from Liwen Bianji, Edanz Editing China (www.liwenbianji.cn/ac), for editing the English text of a draft of this manuscript.

Funding Information This study was funded by the Key Special Project of Pudong New Area Health System (grant no. PWZzk2017-23) and the Foundation for Young Scholars of Shanghai Gongli Hospital of Secondary Military Medical University (grant no. 2018YQNJJ-15).

\section{Compliance with Ethical Standards}

Ethical Approval of Human Studies All procedures performed in this study involving human participants were in accordance with the ethical standards of the institutional research committee and with the 1964 Helsinki declaration and its later amendments. Ethical approval was obtained from the Medicine Ethics Committee of Shanghai Gongli Hospital and registered in the Chinese Clinical Trial Registry (KY2018-168). No animal studies were carried out by the authors for this article.

Conflict of Interest The authors declare that they have no conflict of interest.

Open Access This article is licensed under a Creative Commons Attribution 4.0 International License, which permits use, sharing, adaptation, distribution and reproduction in any medium or format, as long as you give appropriate credit to the original author(s) and the source, provide a link to the Creative Commons licence, and indicate if changes were made. The images or other third party material in this article are included in the article's Creative Commons licence, unless indicated otherwise in a credit line to the material. If material is not included in the article's Creative Commons licence and your intended use is not permitted by statutory regulation or exceeds the permitted use, you will need to obtain permission directly from the copyright holder. To view a copy of this licence, visit http://creativecommons.org/licenses/by/4.0/.

\section{References}

1. Keeley, E. C., Boura, J. A., \& Grines, C. L. (2003). Primary angioplasty versus intravenous thrombolytic therapy for acute myocardial infarction: a quantitative review of 23 randomised trials. Lancet (London, England), 361(9351), 13-20.

2. Wang, H., Zhao, T., Wei, X., Lu, H., \& Lin, X. (2019). The prevalence of 30-day readmission after acute myocardial infarction: a systematic review and meta-analysis. Clinical Cardiology, 42(10), 889-898.

3. Bahit, M. C., Kochar, A., \& Granger, C. B. (2018). Post-myocardial infarction heart failure. JACC. Heart Failure, 6(3), 179-186.

4. Hausenloy, D. J., Bøtker, H. E., Ferdinandy, P., Heusch, G., Ng, G. A., Redington, A., et al. (2019). Cardiac innervation in acute myocardial ischaemia/reperfusion injury and cardioprotection. Cardiovascular Research, 115(7), 1167-1177.

5. Yang, C.-F., Chen, Y.-Y., Singh, J. P., Hsu, S.-F., Liu, Y.-W., Yang, C.-Y., et al. (2019). targeting protein tyrosine phosphatase PTP-PEST for therapeutic intervention in acute myocardial infarction. Cardiovascular Research, cvz165. https://doi.org/10.1093/ cvr/cvzl65.

6. Xu, T., Ding, W., Ao, X., et al. (2019). ARC regulates programmed necrosis and myocardial ischemia/reperfusion injury through the inhibition of mPTP opening. Redox Biology, 20, 414- 426.

7. Sun, T., Ding, W., Xu, T., Ao, X., Yu, T., Li, M., et al. (2019). Parkin regulates programmed necrosis and myocardial ischemia/ reperfusion injury by targeting cyclophilin-D. Antioxidants \& Redox Signaling, 31(16), 1177-1193.

8. Yang, C.-F. (2018). Clinical manifestations and basic mechanisms of myocardial ischemia/reperfusion injury. Ci ji yi xue za zhi $=T z u$ chi Medical Journal, 30(4), 209-215.

9. Quinn, J. J., \& Chang, H. Y. (2016). Unique features of long noncoding RNA biogenesis and function. Nature reviews. Genetics, 17(1), 47-62.

10. Derrien, T., \& Guigó, R. (2011). Long non-coding RNAs with enhancer-like function in human cells. Medecine Sciences, 27(4), 359-361.

11. Jiang, X., \& Ning, Q. (2015). The emerging roles of long noncoding RNAs in common cardiovascular diseases. Hypertension Research: Official Journal of the Japanese Society of Hypertension, 38(6), 375-379.

12. Huang, Y. (2018). The novel regulatory role of lncRNA-miRNAmRNA axis in cardiovascular diseases. Journal of Cellular and Molecular Medicine, 22(12), 5768-5775.

13. Xu, S., Kamato, D., Little, P. J., Nakagawa, S., Pelisek, J., \& Jin, Z. G. (2019). Targeting epigenetics and non-coding RNAs in atherosclerosis: from mechanisms to therapeutics. Pharmacology \& Therapeutics, 196, 15-43.

14. Wang, K., Liu, F., Liu, C. Y., et al. (2016). The long noncoding RNA NRF regulates programmed necrosis and myocardial injury during ischemia and reperfusion by targeting miR-873. Cell Death and Differentiation, 23(8), 1394-1405. https://doi.org/10.1038/ cdd.2016.28.

15. Ibanez, B., James, S., Agewall, S., et al. (2018). 2017 ESC Guidelines for the management of acute myocardial infarction in patients presenting with ST-segment elevation: the Task Force for the management of acute myocardial infarction in patients presenting with ST-segment elevation of the European Society of Cardiology (ESC). European Heart Journal, 39(2), 119-177.

16. Ponikowski, P., Voors, A. A., Anker, S. D., et al. (2016). 2016 ESC Guidelines for the diagnosis and treatment of acute and chronic heart failure. Revista Española de Cardiología (English Edition), 69(12), 1167.

17. Chen, J., Hsieh, A. F., Dharmarajan, K., et al. (2016). National trends in heart failure hospitalization after acute myocardial infarction for Medicare beneficiaries: 1998-2010. Circulation, 128(24), 2577-2584.

18. Weir, R. A. P., McMurray, J. J. V., \& Velazquez, E. J. (2006). Epidemiology of heart failure and left ventricular systolic dysfunction after acute myocardial infarction: prevalence, clinical 
characteristics, and prognostic importance. The American Journal of Cardiology, 97(10A), 13F-25F.

19. Hung, J., Teng, T. H., Finn, J., et al. (2023). Trends from 1996 to 2007 in incidence and mortality outcomes of heart failure after acute myocardial infarction: a population-based study of 20,812 patients with first acute myocardial infarction in Western Australia. Journal of the American Heart Association, 2(5), e000172.

20. Lin, X., Zhang, S., \& Huo, Z. (2019). Serum circulating miR-150 is a predictor of post-acute myocardial infarction heart failure. International Heart Journal, 60(2), 280-286.

21. Tao, H., Yang, J.-J., Hu, W., Shi, K.-H., Deng, Z.-Y., \& Li, J. (2016). Noncoding RNA as regulators of cardiac fibrosis: current insight and the road ahead. Pflügers Archiv / European Journal of Physiology, 468(6), 1103-1111.

22. Leeper, N. J., \& Maegdefessel, L. (2018). Non-coding RNAs: key regulators of smooth muscle cell fate in vascular disease. Cardiovascular Research, 114(4), 611-621.

23. Leung, A., \& Natarajan, R. (2014). Noncoding RNAs in vascular disease. Current Opinion in Cardiology, 29(3), 199-206.

24. Busch, A., Eken, S. M., \& Maegdefessel, L. (2016). Prospective and therapeutic screening value of non-coding RNA as biomarkers in cardiovascular disease. Annals of Translational Medicine, 4(12), 236-236.

25. Araszkiewicz, A., Janus, M., Prech, M., et al. (2014). Relations of diabetes mellitus, microvascular reperfusion and left ventricular remodelling in patients with acute myocardial infarction treated with primary coronary intervention. Kardiologia Polska, 72(1), 20-26.

26. Felker, G. M., Mentz, R. J., Teerlink, J. R., et al. (2015). Serial high sensitivity cardiac troponin $\mathrm{T}$ measurement in acute heart failure: insights from the RELAX-AHF study. European Journal of Heart Failure, 17(12), 1262-1270.

27. Aimo, A., Januzzi Jr., J. L., Vergaro, G., et al. (2018). Prognostic value of high-sensitivity troponin $\mathrm{T}$ in chronic heart failure: an individual patient data meta-analysis. Circulation, 137(3), 286297.

Publisher's Note Springer Nature remains neutral with regard to jurisdictional claims in published maps and institutional affiliations. 